Chirurg 2016 $87: 613$

DOI 10.1007/s00104-016-0225-y

Online publiziert: 13. Juni 2016

๑) Springer-Verlag Berlin Heidelberg 2016

CrossMark

\author{
P. Mroczkowski ${ }^{1}$ C. J. Bruns ${ }^{2}$ \\ ${ }^{1}$ Klinik für Allgemein-, Viszeral- und Gefäßchirurgie, Otto-von-Guericke-Universität Magdeburg, \\ Magdeburg, Deutschland \\ ${ }^{2}$ Klinik und Poliklinik für Allgemein-, Viszeral- und Tumorchirurgie, Universitätsklinikum Köln (AöR), Köln, \\ Deutschland
}

\title{
Liver-first-Option bei simultaner Lebermetastasierung eines kolorektalen Karzinoms
}

CSS voraussagt. Im zweiten Teil der Untersuchung erfolgte eine Match-pairedAnalyse nach Anpassung des BPI.

Welsh FKS, Chandrakumaran K, John TG et al (2016) Propensity score-matched outcomes analysis of the liver-first approach for synchronous colorectal liver metastases. $\mathrm{Br} \mathrm{J}$ Surg doi:10.1002/bjs.10099.

Hintergrund und Fragestellung. Etwa $20 \%$ der Patienten mit einem kolorektalen Karzinom zeigen bereits bei der Primärdiagnose eine Lebermetastasierung. Die Festlegung eines geeigneten Therapieplanes wird kontrovers diskutiert, wobei drei Grundsatzkonzepte zu unterscheiden sind: „liver-first“, „bowel-first“ oder eine Simultanoperation, in der Kombination mit verschiedenen neo-bzw. adjuvanten Chemotherapie schemata. Die vorliegende Arbeit vergleicht monozentrisch Ergebnisse einer primären Leberresektion gefolgt von einer Darmresektion mit einer primären Darmresektion gefolgt von einer Leberresektion.

Methoden. Verglichen wurden Überlebensdaten bei Median-Follow-up von 34 Monaten: Gesamtüberleben (OS), tumorspezifisches Überleben (CSS), tumorfreies Überleben (DFS). Bestimmt wurde auch der Basingstoke Prediction Index (BPI), der auf der Grundlage von Lymphknotenstatus und Differenzierungsgrad des Primarius, CEA-Spiegel zum Zeitpunkt der Leberresektion, Anzahl und Größe der Lebermetastasen, R-Status der Leberresektion und extrahepatischen Tumormanifestationen das
Ergebnisse. Bei 98 Patienten erfolgte primär eine Leberresektion, bei 467 eine Darmresektion. Die erste Gruppe zeigte einen signifikant höheren BPI: 8,5 vs. 8 $(p=0,030)$ und ein schlechteres 5 Jahres-DFS: $23 \%$ vs. $45,6 \%$ ( $p=0,001)$, die Unterschiede im OS sowie CSS waren nicht signifikant. In der Matched-pairedAnalyse wurden keine Überlebensunterschiede festgestellt.

Diskussion und Fazit. In der vorliegenden Studie gab es eine negative Selektion (mehr fortgeschrittene Tumoren mit ungünstigerer Prognose) für die primäre Leberoperation. So verwundert das schlechtere Outcome vor dem Matching nicht. Die Gleichwertigkeit der beiden Methoden, sowie in ausgewählten Fällen einer simultanen Operation, im Einklang mit einer kürzlich publizierten Literaturauswertung [1] erlaubt eine individuelle Anpassung der Behandlungsstrategie. Weiterhin sollte zuerst die Lokalisation operiert werden, die am meisten von einer lokalen Inoperabilität bedroht ist. Da die Patienten auch eine systemische Therapie vor, nach oder zwischen den Eingriffen erhalten sollen [2], ist eine interdisziplinäre Abstimmung des Vorgehens wichtig. Chirurgische Komplikationen - Wundheilungsstörungen, Biliome, Abszesse - dürfen nicht die Verabreichung der Chemotherapie verzögern, auf der anderen Seite dürfen die Nebenwirkungen der Chemotherapie das Risikoprofil für die Operation nicht gefährden. Bei der Vielfalt der Konstellationen ist hier aktuell keine saubere randomisierte klinische Studie zu erwarten.

\section{Korrespondenzadresse}

\section{P. Mroczkowski}

Klinik für Allgemein-, Viszeral- und Gefäßchirurgie, Otto-von-Guericke-Universität Magdeburg

Leipziger Straße 44, 39120 Magdeburg,

Deutschland

pawel.mroczkowski@med.ovgu.de

Interessenkonflikt. P. Mroczkowski und C. J. Bruns geben an, dass kein Interessenkonflikt besteht.

\section{Literatur}

1. Baltatzis M, Chan AK, JegatheeswaranS, Mason JM Siriwardena AK (2016) Colorectal cancer with synchronous hepatic metastases: Systematic review of reports comparing synchronous surgery with sequential bowel-first or liver-first approaches. Eur JSurg Oncol 42(2):159-165

2. Velde CJ van de, Boelens PG, Borras JM, Coebergh JW, Cervantes A, Blomqvist L et al (2014) EURECCA colorectal: Multidisciplinary management: European consensus conference colon \& rectum. Eur JCancer 50(1):1 